\title{
Intelligent control and monitoring system to improve security in nuclear power plants
}

\author{
Vorobioff $\mathrm{J}^{1,3}$, Boggio N. ${ }^{1,2,3}$, Checozzi F. R. ${ }^{1}$ and Rinaldi C. ${ }^{1,2,3}$ \\ ${ }^{1}$ CNEA, Av. Gral Paz 1499, San Martín, Bs.As. Argentina \\ ${ }^{2}$ CONICET, Godoy Cruz 2290, C.A.B.A., Argentina \\ ${ }^{3}$ Universidad Tecnológica Nacional, Sarmiento 440, C.A.B.A., Argentina \\ ${ }^{4}$ Universidad Nacional de San Martín, 25 de Mayo y Francia, San Martín, Bs.As. Argentina \\ vorobioff@cnea.gov.ar
}

\begin{abstract}
Many nuclear plants exceed their lifetimes, have obsolete security systems and cannot be considered failsafe. In this work the implementation of an intelligent system of telemetry and control is proposed to improve security at nuclear plants. These systems can be added to current systems or replace the existing ones. With simple remote sensing devices, low cost, easy installation and wireless communication or wiring, depending on the control area, security at nuclear plants can be improved. Remote devices, fixed and mobile, send information to a central computer with an intelligent control system. The mobile devices, using suitable algorithms will move and seek greater risk areas: high levels of radiation, high concentrations of contaminants or explosive gases, high temperatures, presence in prohibited zones, etc. Devices more sophisticated such as cameras and audio acquisition systems can also be implemented. The central computer will process the data using regression algorithms, pattern recognition and artificial intelligence. These algorithms have intelligent learning, detecting risks and generating alerts in real time. The learning system interacts with the operator to establish optimum processing parameters, rejecting false alarms. Simple graphics were used to display the current state of the plant and trend graphs to predict accidents. Also, mobile devices may be carried by the operator, by a simple and small robot or by a conveyor belt. The devices will be strategically located in confined spaces and highly dangerous areas to monitor the machines. These implementations will reduce the risk of accidents and improve preventive maintenance of nuclear plants.
\end{abstract}

Key words: gas sensors, nuclear plants, security, algorithms, pattern recognition

\section{Introduction}

The proposed work consists of a telemetry and intelligent control system for safety improvements on nuclear plants. Nuclear energy plants are some of the most sophisticated systems ever designed [1]. Any complex system, no matter how well designed and built be, can fail [2].

Many nuclear plants exceed their lifetimes, have obsolete security systems and may fail in many ways. Security risks may be higher when nuclear systems are newer, and operators have less experience using them. At the same time, routine work and high workload during plant operation cause a bigger accident risk [3].

In the last few years, embedded measurement systems progressed notably, size and costs were reduced, and features were improved. Nevertheless, it's needed to process the obtained information. In the present work it is proposed to install intelligent and redundant systems to improve security.

\section{Methods}

In this work, the Raspberry PI minicomputer, model 3 coupled to a thin film MOS sensor is used. It employs a Linux operative system, and communicates via Ethernet and $\mathrm{Wi}-\mathrm{Fi}$, generating a widely flexible data server, with reduced size and simple communication through PC, smartphones and tablets. Its cost is less than U\$S 100 and it has an HDMI output for on-screen visualization. See Fig. 1, minicomputer with sensors chamber. 


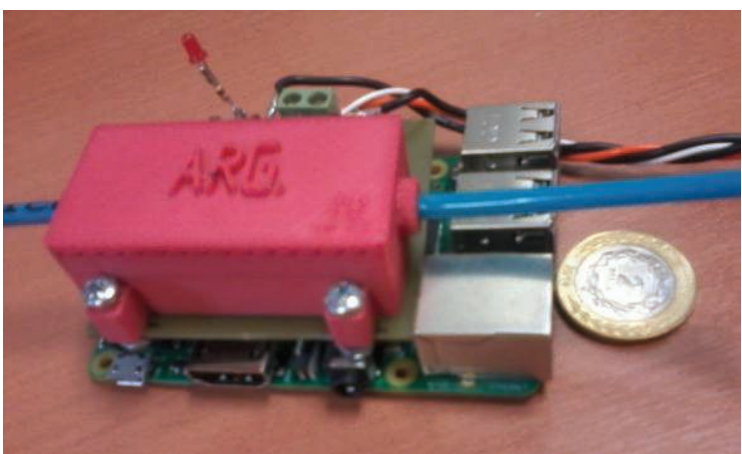

Figure 1.Fixed measurement system implemented using Gas Sensors and Raspberry pi minicomputer

As mobile sensing device, a mobile robot, model Elegoo Arduino kit 006, is used with added sensors, see Fig 2. Using proper algorithms, it moves and searches riskier zones: gas leaks, high concentrations of contaminant and/or explosive gases, high temperatures, entering in prohibited places, etc.

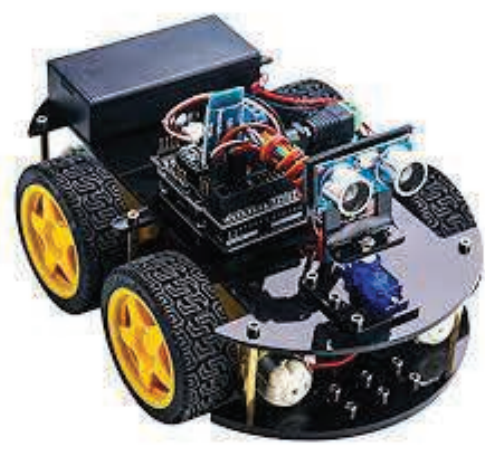

Figure 2.Mobile measurement system implemented using a model Elegoo Arduino kit 006 mobile robot

It's proposed that a central computer receives all the information, stores and processes data employing regression calculation, pattern recognition and artificial intelligence algorithms $[4,5]$. These algorithms will be based on Computational Intelligence, recognizing behavior through intelligent learning, detecting threats and generating in turn opportune real time alerts. The learning system will interact with the operator to adjust optimal processing parameters, discarding false alarms. Simple graphics will be used to visualize the actual state of the installations and tendency graphs to predict possible accidents too.

The proposed project proposes the implementation of distinct solutions according to the requirements of the nuclear plant. These solutions can be reliable, low cost and easy to install. The information will be analyzed correctly, for this purpose, different algorithms will be implemented. The algorithms will recognize plant behavior via intelligent learning, classifying the relevant data and sending alarms of different levels to cell phones and computers. The information must be properly separated to comply with the regulations of the nuclear plants. Only information about possible risks will be sent, establishing different levels of computer security and hiding secret information from the plant.

\section{Results and Conclusions}

Many gas, temperature, and humidity sensors, flowmeters, mass flow controllers and actuators were tested, getting satisfactory results. Signal conditioning and data transmission electronics were designed and installed. As an example, in Fig. 3 the response of $\mathrm{SnO}_{2}$ sensors exposed to $\mathrm{CO}$ is shown.

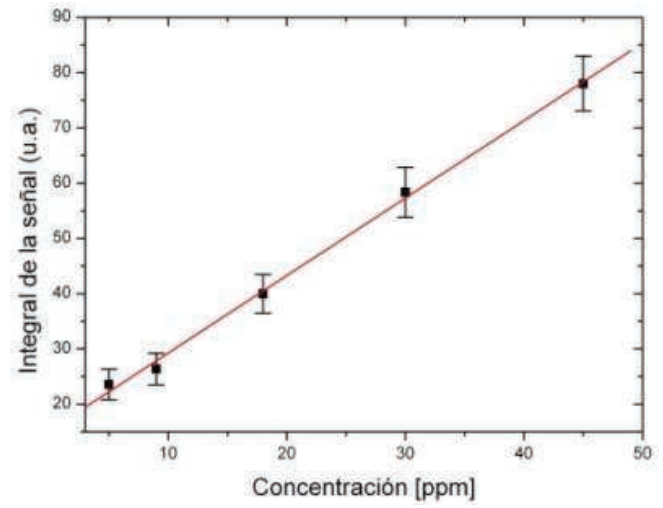

Figure 3. $\mathrm{SnO}_{2}$ sensors response. Signal Integral for different concentrations of CO. ( $)$ ) Average value, (-) Linear fit.

It's expected to reduce accident risks caused by human error, technical failure or unexpected situations.

As an example, this system can be implemented in the food irradiation plant with gamma rays, where people cannot access.

\section{References}

[1] Jan Willem Storm van Leeuwen (2008). Nuclear power - the energy balance

[2] François Diaz Maurin (2011). Fukushima: Consequences of Systemic Problems in Nuclear Plant Design, Economic \& Political Weekly (Mumbai) Vol. 46, No. 13, pp.10-12, 26 March, 2011.

[3] M.V. Ramana. Nuclear Power: Economic, Safety, Health, and Environmental Issues of Near-Term Technologies, Annual Review of Environment and Resources, 2009. 34, pp.139-140.

[4] Webb, A.R. and Copsey K.D. Statistical Pattern Recognition,.3ed, Wiley, 1-4, 2011.

[5] Scott, S. M., James, D., Ali, Z. Data analysis for electronic nose systems. Microchim. Acta 2006, 156, 183, 2006. 